\title{
T1期乳腺癌患者发生同侧腋窝淋巴结转移风险 列线图的建立
}

付媛媛 ${ }^{1,2}$, 姜晶釒金, 陈述政 ${ }^{2}$, 邱福铭 ${ }^{1}$

1. 浙江大学医学院附属第二医院肿瘤内科,浙江杭州 310009

2. 浙江大学丽水医院乳腺外科, 浙江 丽水 323000

[摘要] 目的:建立 $\mathrm{T} 1$ 期 (原发肿瘤最大直径 $2 \mathrm{~cm}$ 及以下)乳腺癌患者发生同侧 腋窝淋巴结转移风险的列线图。方法: 收集 2010 年 1 月至 2015 年 6 月在浙江大学 医学院附属第二医院及浙江大学丽水医院接受手术治疗的 $\mathrm{T} 1$ 期乳腺癌患者的临床 病理资料。共入组 907 例患者, 其中浙江大学医学院附属第二医院患者作为建模组 $(n=573)$, 浙江大学丽水医院患者作为验证组 $(n=334)$ 。运用单因素 Logistic 回归分 析风险因素, 多因素Logistic 回归进一步篎选独立影响因素, 利用影响因素建立预 测 T1 期乳腺癌患者同侧腋窝淋巴结转移风险的列线图。运用 $\mathrm{C}$ 指数、受试者操作 特征曲线、校准曲线以及临床决策曲线分析模型的校准度、预测能力和临床效 益。结果: 单因素分析结果显示, T1 期乳腺癌患者发生同侧腋窝淋巴结转移与原发 肿瘤大小、脉管癌栓、Ki-67、组织病理学分级和分子分型相关 $(P<0.05$ 或 $P<0.01)$ 。多因素Logistic 回归分析显示, T1 期乳腺癌患者发生同侧腋窝淋巴结转 移的独立影响因素为原发肿瘤大于 $0.5 \mathrm{~cm}$ 、有脉管癌栓、Ki-67 阳性、雌激素受体 (ER) 阳性以及组织病理学分级 $2 \sim 3$ 级 $(P<0.05$ 或 $P<0.01)$ 。基于上述 5 个独立影 响因素构建列线图预测模型，建模组和验证组 C 指数分别为 $0.739(95 \% \mathrm{CI}$ : 0.693 0.785)和 0.736(95\% CI:0.678 0.793), 模型预测能力良好。建模组和验证 组校正曲线、临床决策曲线提示模型一致性和临床获益良好。结论: 原发肿瘤大 小、组织病理学分级、脉管癌栓、Ki-67 和 ER 状态是 T1 期乳腺癌患者发生同侧 腋窝淋巴结转移的重要预测因素。建立的风险预测列线图可以有效预测患者发生 同侧腋窝淋巴结转移的风险, 为临床医生制订个体化的腋窝管理方案提供参考。

[关键词] 乳腺肿瘤; 淋巴转移; 危险因素; 列线图; 预测

[中图分类号 ] R730.4 [文献标志码 ] A

\section{Establishment of risk prediction nomogram for ipsilateral axillary lymph node metastasis in $\mathrm{T} 1$ breast cancer}

FU Yuanyuan ${ }^{1,2}$, JIANG Jingxin ${ }^{1}$, CHEN Shuzheng ${ }^{2}$, QIU Fuming ${ }^{1}$ (1. Department of

收稿日期: 2020-11-30 接受日期:2020-12-24

基金项目:国家自然科学基金(81672802); 浙江省自然科学基金(LZ17H160004)

第一作者:付媛媛,主治医师,主要从事乳腺癌临床研究;E-mail :zjdrfu@163. com; https ://orcid. org/0000-0001-6144-4789 通信作者: 邱福铭,主任医师,博士生导师,主要从事乳腺癌、大肠癌等基础和临床治疗; E-mail :zdf2zlk@163.com; https://orcid. org/0000-0001-5793-1023 
Oncology,the Second Affiliated Hospital,Zhejiang University School of Medicine, Hangzhou 310009, China; 2. Department of Breast Surgery, Zhejiang University Lishui Hospital,Lishui 323000, Zhejiang Province, China)

Corresponding author:QIU Fuming,E-mail:zdf2zlk@163.com,https://orcid.org/00000001-5793-1023

[Abstract] Objective:To establish and verify a risk prediction nomogram for ipsilateral axillary lymph node metastasis in breast cancer stage T1 (mass $\leqslant 2 \mathrm{~cm}$ ). Methods: The clinicopathological data of 907 patients with T1 breast cancer who underwent surgical treatment from January 2010 to June 2015 were collected, including 573 cases from the Second Affiliated Hospital of Zhejiang University School of Medicine (modeling group) and 334 cases from Zhejiang University Lishui Hospital (verification group). The risk factors of ipsilateral axillary lymph node metastasis were analyzed by univariate and multivariate logistic regression. The influencing factors were used to establish a nomogram for predicting ipsilateral axillary lymph nodes metastasis in $\mathrm{T} 1$ breast cancer. The model calibration, predictive ability and clinical benefit in the modeling group and the verification group were analyzed by $\mathrm{C}$ index, receiver operating characteristic curve,calibration curve and decision curve analysis (DCA) curve, respectively. Results: Univariate analysis showed that lymph node metastasis was related with primary tumor size, vascular tumor thrombus , Ki-67, histopathological grade, and molecular type $(P<0.05$ or $P<0.01)$. Multivariate logistic regression analysis showed that the primary tumor $>0.5 \mathrm{~cm}$, vascular tumor thrombus, Ki-67 positive, estrogen receptor (ER) positive, and histopathological grade 2-3 were independent risk factors of axillary lymph node metastasis $(P<0.05$ or $P<0.01)$. Based on the independent risk factors, a nomogram prediction model was established. The $\mathrm{C}$ indexes of the model group and the validation group were 0.739 (95\%CI:0.693-0.785) and 0.736 (95\%CI:0.678-0.793), respectively. The calibration curve and DCA curve of the modeling group and the verification group indicated that the model was consistent and had good clinical benefit. Conclusions : Primary tumor size, histopathological grade, vascular tumor thrombus, Ki-67, and ER status are predictors of ipsilateral axillary lymph node metastasis in T1 breast cancer. The established prediction nomogram can effectively predict the risk of ipsilateral axillary lymph node metastasis in T1 breast cancer, which can be used as a reference for individualized axillary management.

[Key words ] Breast neoplasm; Lymphatic metastasis; Risk factors; Nomogram; Prediction

[ J Zhejiang Univ (Med Sci), 2021, 50(1): 81-89. ]

[缩略语] 腋窝淋巴结清扫 (axillary lymph node dissection, ALND); 前哨淋巴结活检 (sentinel lymph node biopsy,SLNB); 雌激素受体 (estrogen receptor,ER); 孕激素受体 (progesterone receptor,PR); 人表皮生长因子受体2(human epithelial growth factor receptor-2,Her-2)；受试者操作特征曲线 (receiver operating characteristic curve, ROC 曲线) 
流行病学资料显示, 乳腺癌位于我国女性恶 性肿瘤发病率的首位,2015 年我国乳腺癌新发人 数约为 30.4 万, 约占女性所有新发恶性肿瘤的 $17.10 \%$, 发病率为 $45.29 / 10$ 万 $^{[1]}$ 。目前我国乳腺 癌患者 5 年存活率高达 $83.2 \%$, 早期乳腺癌尤其 是 $\mathrm{T} 1$ 期 (原发肿瘤最大直径 $2 \mathrm{~cm}$ 及以下) 乳腺癌 患者拥有更长的存活期, 因此其对术后生活质量 要求更高。目前, 早期乳腺癌患者ALND术后并发 症主要有上肢淋巴水肿、疼痛、肩关节活动障碍、 手术侧肢体麻木等 ${ }^{[2]}$ 。许多临床研究发现, 早期 乳腺癌患者进行 SLNB 与行 ALND 后肿瘤局部复 发率及患者存活率差异并无统计学意义 ${ }^{[3-5]}$, 所以 在治疗上 SLNB 逐步增多。但 SLNB 仍然是有创 性操作, 为进一步减少术后并发症, 诸多研究者在 探索对于早期乳腺癌患者免除 SLNB 的可行性, 如 对早期乳腺癌患者同侧腋窝淋巴结进行细针穿刺 明确转移情况或通过放射组学来预测腋窝淋巴结 状态 ${ }^{[6-8]}$ 。本研究将通过分析 907 例 $\mathrm{T} 1$ 期乳腺癌 患者的相关临床病理学特征与同侧腋窝淋巴结转 移的关系, 寻找 T1 期乳腺癌患者发生同侧腋窝淋 巴结转移的危险因素, 并建立风险预测列线图篮 查和识别淋巴结转移患者, 为临床医生制订个体 化的腋窝管理方案提供参考。

\section{1 资料与方法}

\section{1 资料来源}

收集 2010 年 1 月至 2015 年 6 月在浙江大学 医学院附属第二医院 (简称浙医二院) 及浙江大学 丽水医院(简称丽水医院)首次接受手术治疗且临
床资料完整的 $\mathrm{T} 1$ 期乳腺癌患者。排除标准: (1)双 侧乳腺癌患者; (2)新辅助化疗后或既往因其他恶 性肿瘤接受过放化疗治疗者; (3)Tis 原位癌(包括 小叶原位癌、导管原位癌及佩吉特病)、微小浸润 性乳腺癌(Tmic)患者; (4)病理资料缺失、原发肿瘤 大小无法评估或腋窝淋巴结转移数量未评估 者; (5)男性患者 (男性患者占比极小)。从2537例 患者中篮选出符合条件的患者共 907 例, 篮选流 程见图 1 。其中, 573 例来自浙医二院的患者作为 建模组,334 例来自丽水医院的患者作为验证组。 收集患者的临床及病理特征,包括年龄,原发肿瘤 大小 ( $\mathrm{T}$ 分期), 组织病理学分级, 是否伴有脉管癌 栓, 是否有导管原位癌成分, ER、PR、Her-2、Ki67 表达情况, 分子分型及腋窝淋巴结情况。建模 组和验证组年龄、原发肿瘤大小、导管原位癌成 分、ER、PR、Ki-67、分子分型以及组织病理学分级 差异存在统计学意义 $(P<0.05$ 或 $P<0.01$, 表 1$)$, 符 合来自不同临床中心样本的差异, 如果建模组建 立的模型在验证组中获得可靠的预测价值,将进 一步证明模型的适用性好。

\section{2 乳腺癌的 $\mathrm{T}$ 分期}

肿瘤大小以术后大体标本中的病变范围计 算。参照美国癌症联合会 $(\mathrm{AJCC})$ 第 8 版 ${ }^{[9]}$,本研 究根据浸润灶的最大直径将 $\mathrm{T} 1$ 期患者分为 3 组: 最大直径介于 $0.1 \sim 0.5 \mathrm{~cm}$ 为 $\mathrm{T} 1 \mathrm{a},>0.5 \sim 1.0 \mathrm{~cm}$ 为 $\mathrm{T} 1 \mathrm{~b},>1.0 \sim 2.0 \mathrm{~cm}$ 为 $\mathrm{T} 1 \mathrm{c}$ 。

\section{3 免疫组织化学检测确定乳腺癌的分子分型} 通过免疫组织化学检测对患者的 $E R 、 P R$ 、 Her-2、Ki-67 进行评估, ER、PR $\geqslant 1 \%$ 为阳性, Ki-67

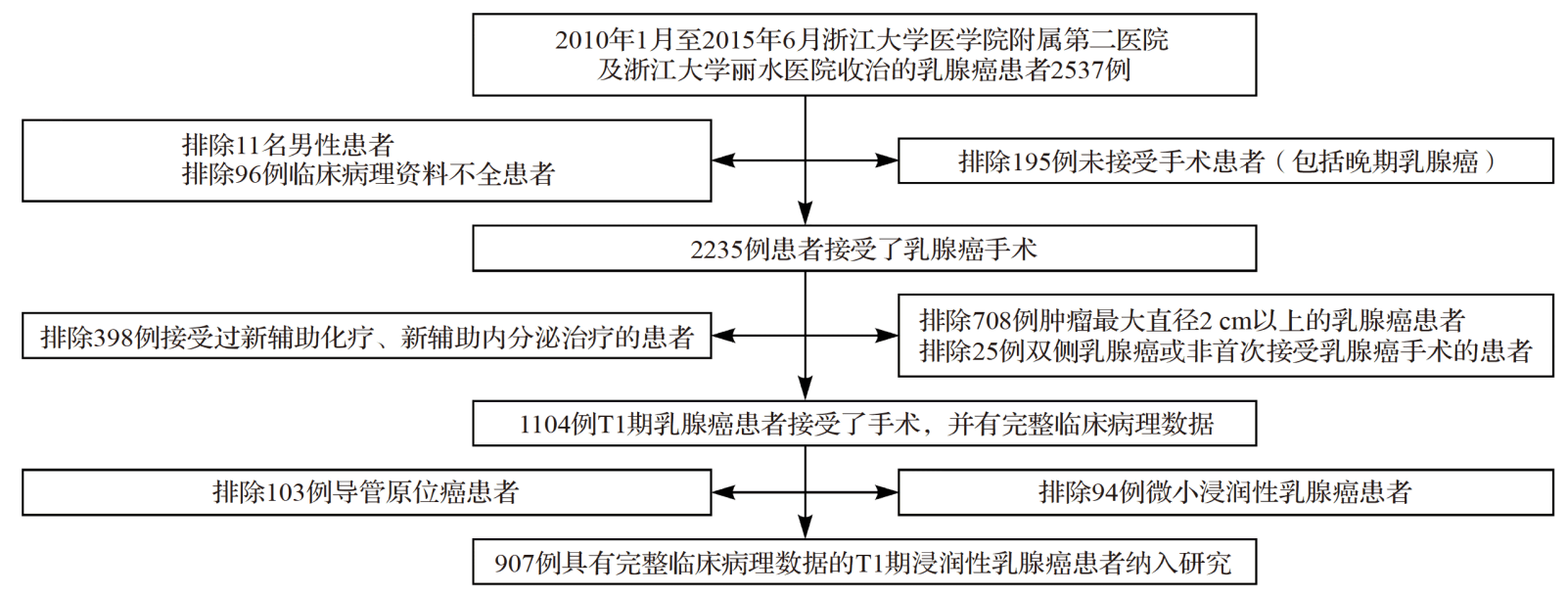

图 1 研究对象篮选流程图

Figure 1 Research objects screening flowchart 
表 1 建模组与验证组临床和病理基线特征比较

Table 1 Baseline characteristics of the patients in the modeling group and validation group $[n(\%)$ 或 $\bar{x} \pm s]$

\begin{tabular}{|c|c|c|c|c|c|c|c|c|c|c|c|}
\hline \multirow{2}{*}{ 组别 } & \multirow{2}{*}{$n$} & \multirow{2}{*}{$\begin{array}{l}\text { 年龄 } \\
50 \text { 岁* }\end{array}$} & \multirow{2}{*}{$\begin{array}{c}\text { 淋巴结 } \\
\text { 转移 }\end{array}$} & \multicolumn{3}{|c|}{$\mathrm{T}$ 分期 } & \multirow{2}{*}{$\begin{array}{l}\text { 有脉管 } \\
\text { 癌栓 }\end{array}$} & \multirow{2}{*}{$\begin{array}{l}\text { 有导管原 } \\
\text { 位癌成分 }\end{array}$} & \multirow{2}{*}{ ER 阳性 } & \multirow{2}{*}{ PR 阳性 } & \multirow{2}{*}{ Her-2 阳性 } \\
\hline & & & & $\mathrm{T} 1 \mathrm{a}$ & $\mathrm{T} 1 \mathrm{~b}$ & $\mathrm{~T} 1 \mathrm{c}$ & & & & & \\
\hline 建模组 & 573 & $319(55.7)$ & $141(24.6)$ & $47(8.2)$ & $114(19.9)$ & $412(71.9)$ & $44(7.7)$ & $174(30.4)$ & $408(71.2)$ & $357(62.3)$ & $134(23.4)$ \\
\hline 验证组 & 334 & $175(52.5)$ & $97(29.0)$ & $31(9.3)$ & $44(13.2)$ & $259(77.5)$ & $35(10.5)$ & $44(13.2)$ & $263(78.7)$ & $236(70.7)$ & $79(23.7)$ \\
\hline$t / \chi^{2}$ 值 & - & 5.509 & 249.161 & & 6.666 & & 2. 081 & 34.161 & 6.229 & 6.508 & 0.008 \\
\hline$P$ 值 & - & $<0.05$ & $<0.01$ & & $<0.05$ & & 0.149 & $<0.01$ & $<0.05$ & $<0.05$ & 0.927 \\
\hline \multirow[b]{2}{*}{ 组别 } & \multirow[b]{2}{*}{$n$} & \multirow[b]{2}{*}{ Ki-67 阳性 } & \multicolumn{4}{|c|}{ 组织病理学分级 } & \multicolumn{5}{|c|}{ 分子分型 } \\
\hline & & & 1 级 & 2 级 & 3 级 & 未分级 & $\begin{array}{c}\text { Luminal } \\
\text { A 型 }\end{array}$ & $\begin{array}{c}\text { Luminal B } \\
\text { 型(Her-2 } \\
\text { 阴性) }\end{array}$ & $\begin{array}{c}\text { Luminal B } \\
\text { 型(Her-2 } \\
\text { 阳性) }\end{array}$ & $\begin{array}{l}\text { Her-2 过 } \\
\text { 表达型 }\end{array}$ & $\begin{array}{l}\text { 三阴性 } \\
\text { 乳腺癌 }\end{array}$ \\
\hline 建模组 & 573 & $279(48.7)$ & $110(19.2)$ & $312(54.5)$ & $124(21.6)$ & $27(4.7)$ & $229(40.0)$ & $123(21.5)$ & $68(11.9)$ & $66(11.5)$ & $87(15.2)$ \\
\hline 验证组 & 334 & $204(61.1)$ & $44(13.2)$ & $145(43.3)$ & $122(36.5)$ & $23(6.9)$ & $114(34.1)$ & 104(31. 1) & $47(14.1)$ & $32(9.6)$ & $37(11.1)$ \\
\hline$t / \chi^{2}$ 值 & - & 13.005 & \multicolumn{4}{|c|}{28.660} & \multicolumn{5}{|c|}{13.928} \\
\hline$P$ 值 & - & $<0.01$ & \multicolumn{4}{|c|}{$<0.01$} & \multicolumn{5}{|c|}{$<0.01$} \\
\hline
\end{tabular}

“_”: 无相关数据. ${ }^{*}$ 考虑到中国女性普遍绝经年龄约为 50 岁,故以 50 岁为分界. ER:雌激素受体;PR: 孕激素受体;Her-2:人表皮生 长因子受体 2 .

指数 $\geqslant 20 \%$ 为阳性 ${ }^{[10]}$ 。Her- 2 状态分为 $0 、 1+, 2+$ 或 $3+$ 。评分 0 或 $1+$ 为 Her-2 阴性, 评分 $3+$ 为 Her-2 阳性, 评分 2+时进一步行苂光原位杂交检测 Her-2 基因。根据 2015 年圣加伦国际乳腺癌会议上提 出的乳腺癌分类标准 ${ }^{[11]}$, 将乳腺癌分为 5 类: Luminal A 型、Luminal B 型(Her-2 阴性)、Luminal $B$ 型 (Her-2 阳性)、Her-2 过表达型和三阴性乳 腺癌。

\section{4 组织病理学分级}

所有浸润性乳腺癌均使用诺丁汉组织学分级 半定量评分表评估, 由形态学特点 (腺管形成的比 例、细胞核的多形性以及核分裂褒计数) 决定。对 每项特点从 1 分 (良好)至 3 分(差)进行评分, 然后 将三类分数相加, 评出三个等级: $3 \sim 5$ 分为 1 级, $6 \sim 7$ 分为 2 级, $8 \sim 9$ 分为 3 级 ${ }^{[12]}$ 。

\section{5 淋巴结状态}

以 SLNB 或 ALNB 结果作为淋巴结分期标准, 根据常规 HE 染色结果判断淋巴结是否受累。转 移灶 $>2 \mathrm{~mm}$ 为宏转移, 转移灶 $>0.2 \sim 2 \mathrm{~mm}$ 为微转 移, 二者均作为腋窝淋巴结转移; 转移灶 $\leqslant 0.2 \mathrm{~mm}$ 的孤立肿瘤细胞视为腋窝淋巴结无转移。

\section{6 统计学方法}

采用SPSS 23.0 软件进行统计学分析。计数 资料用例数和百分比 $[n(\%)]$ 描述, 采用 $\chi^{2}$ 检验, 计
量资料用均数 \pm 标准差 $(\bar{x} \pm s)$ 描述, 采用 $t$ 检验, $P<0.05$ 为差异具有统计学意义。部分临床病理 信息不明确的情况在统计分析中按缺失值处理。 对建模人群进行单因素Logistic 回归分析, 以 $P<0.05$ 为标准纳人多因素 Logistic 回归分析, 采用 后退法,笠选出 $\mathrm{T} 1$ 期乳腺癌发生同侧腋窝淋巴结 转移的独立危险因素。运用独立危险因素 在 RStudio 4. 0.3 中建立列线图, 采用 ROC 曲线、 $\mathrm{C}$ 指数及临界值下的敏感度和特异度评估模型的 区分度,采用 Hosmer-Lemeshow 检验评价模型拟 合度, 采用 ROC 曲线临界值结合临床决策曲线评 价模型的临床有效性。本次研究采用RStudio 4. 0.3 中rms、pROC、forestplot 程序包, 采用 Bootstrap 进行 内外部验证。

\section{2 结 果}

$2.1 \mathrm{~T} 1$ 期乳腺癌患者发生腋窝淋巴结转移的相 关因素分析

Logistic 单因素分析结果显示, 建模组腋窝淋 巴结转移与原发肿瘤大小 ( $\mathrm{T}$ 分期)、脉管癌栓、 $\mathrm{Ki}-$ 67 、分子分型、组织病理学分级相关 $(P<0.05$ 或 $P<0.01)$,见表 2 。将 $\mathrm{ER} 、 \mathrm{PR} 、 \mathrm{Her}-2^{[13-14]}$ 及单因素 分析中有统计学意义的变量纳人多因素分析。 Logistic 多因素回归分析结果显示, 建模组腋窝淋 
巴结转移的独立影响因素为原发肿瘤大于 $0.5 \mathrm{~cm}$ 、有脉管癌栓、Ki-67 阳性、ER 阳性以及组 织病理学分级 $2 \sim 3$ 级 $(P<0.05$ 或 $P<0.01)$, 见 图 2。结果提示, 原发肿瘤大于 $0.5 \mathrm{~cm}$ 、有脉管癌 栓、Ki-67阳性、ER 阳性以及组织病理学分 级 $2 \sim 3$ 级是 $\mathrm{T} 1$ 期乳腺癌发生同侧腋窝淋巴结转 移的独立影响因素。

$2.2 \mathrm{~T} 1$ 期乳腺癌患者发生腋窝淋巴结转移风险 列线图的建立及验证

采用原发肿瘤大小 ( $\mathrm{T}$ 分期)、组织病理学分 级、脉管癌栓、Ki-67、ER 建立列线图 (图 3), 计算 出该模型的 $\mathrm{C}$ 指数为 $0.739(95 \% C I: 0.693$ $0.785)$, 并绘制 ROC 曲线, 得到曲线下面积为 0.739 (图 4A), 说明该模型具有良好的预测性, 其 最佳截取值为 0.2 , 此时模型的敏感度为 $69.7 \%$ 、 特异度为 $66.1 \%$ 。使用 Bootstrap 对模型进行内部 验证, $B=1000$ 进行重复抽样, 获得校正曲线 (图 5A), 三条曲线走向一致且靠拢, 校正曲线表 现良好, 显示列线图模型与理想模型一致性 尚可。

在验证组对模型进行验证, 得到 $\mathrm{C}$ 指数为 $0.736(95 \% C I: 0.678 \sim 0.793)$, 并绘制 ROC 曲线, 得 AUC 值为 0.736 (图 4B)。同样采用 1000 次
表 2 建模组 T1 期乳腺癌临床病理特征与腋窝淋巴结转 移关系的单因素分析结果

Table 2 Results of univariate logistic regression analysis of clinicopathological characteristics and ipsilateral axillary lymph node metastasis in the modeling group

\begin{tabular}{lcc}
\hline \multicolumn{1}{c}{ 影响因素 } & $O R(95 \% C I)$ & $P$ 值 \\
\hline 年龄 & $0.843(0.576 \sim 1.234)$ & $>0.05$ \\
原发肿瘤大小(T 分期) & & \\
T1b & $4.787(1.072 ~ 21.377)$ & $<0.05$ \\
T1c & $0.138(2.182 ~ 38.274)$ & $<0.01$ \\
导管原位癌成分 & $0.803(0.526 ~ 1.227)$ & $>0.05$ \\
脉管癌栓 & $6.442(3.368 \sim 12.322)$ & $<0.01$ \\
ER 阳性 & $1.242(0.807 \sim 1.911)$ & $>0.05$ \\
PR 阳性 & $0.929(0.629 \sim 1.373)$ & $>0.05$ \\
Her-2 阳性 & $1.356(0.879 \sim 2.093)$ & $>0.05$ \\
Ki-67 阳性 & $2.269(1.532 ~ 3.360)$ & $<0.01$ \\
分子分型 & & \\
Luminal B 型(Her-2 阴性) & $2.325(1.414 ~ 3.823)$ & $<0.01$ \\
Luminal B 型(Her-2 阳性) & $1.802(0.971 ~ 3.344)$ & $>0.05$ \\
Her-2 过表达型 & $1.749(0.934 ~ 3.275)$ & $>0.05$ \\
三阴性乳腺癌 & $0.975(0.516 ~ 1.841)$ & $>0.05$ \\
组织病理学分级 & & \\
2 级 & $3.391(1.687 \sim 6.816)$ & $<0.01$ \\
3 级 & $6.316(3.002 ~ 13.287)$ & $<0.01$ \\
未分级 & $1.739(0.501 \sim 6.040)$ & $>0.05$ \\
\hline
\end{tabular}

$\mathrm{ER}$ : 雌激素受体; $\mathrm{PR}$ : 孕激素受体; Her-2 : 人表皮生长因子 受体 2 .

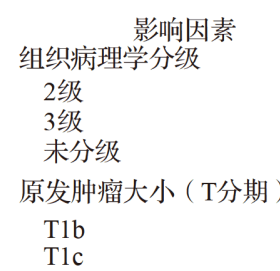

有脉管癌栓

ER阳性

PR阳性

Ki-67阳性

Her-2 阳性

分子分型

Luminal B型 ( Her-2阴性)

Luminal B型（Her-2阳性）

Her-2 过表达型

三阴性乳腺癌

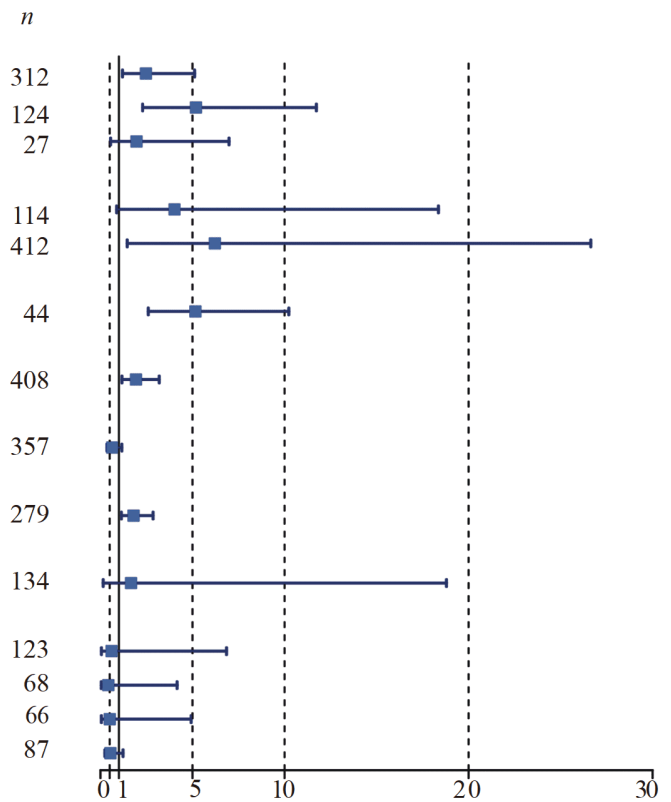

以 T1a、无脉管癌栓、雌激素受体 (ER) 阴性、孕激素受体 (PR) 阴性、人表皮生长因子受体 2(Her-2)阴性、Ki-67 阴性、组织病理学分 级 1 级、Luminal $A$ 型作为基线.

图 2 建模组 $\mathrm{T} 1$ 期乳腺癌患者部分临床病理特征与腋窝淋巴结转移关系的多因素分析结果

Figure 2 Result of multivariate logistic regression analysis of clinicopathological characteristics and ipsilateral axillary lymph node metastasis in the modeling group 


分值
Ki-67

图 $3 \mathrm{~T} 1$ 期乳腺癌患者发生同侧腋窝淋巴结转移的风险列线图

Figure 3 A nomogram of the risk of ipsilateral axillary lymph node metastasis in patients with T1 breast cancer

A

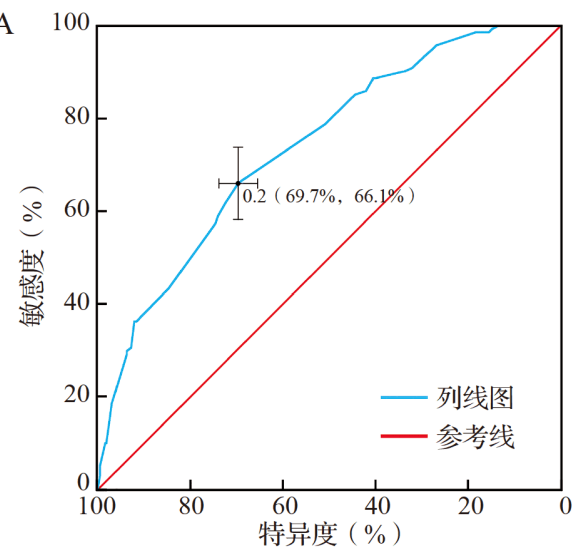

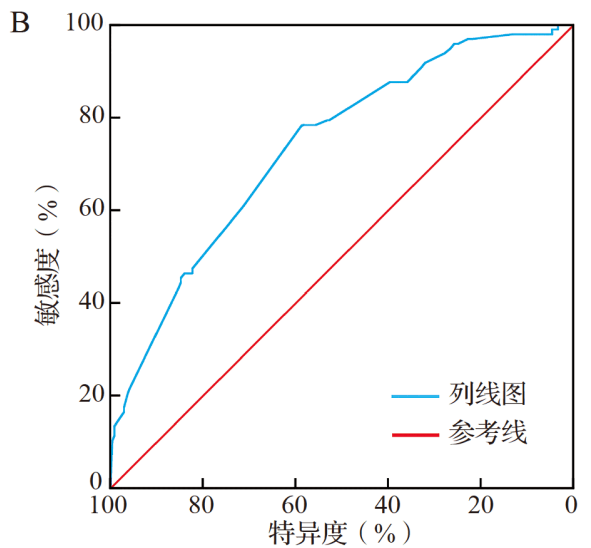

$A$ : 建模组,临界值为 0.2 , 曲线下面积为 0.739 ; B : 验证组,曲线下面积为 0.736 .

图 $4 \mathrm{~T} 1$ 期乳腺癌患者发生同侧腋窝淋巴结转移风险预测模型的 ROC 曲线

Figure 4 ROC analysis of the prediction model of the risk of ipsilateral axillary lymph node metastasis in patients with T1 breast cancer

Bootstrap 重采样, 校正曲线提示预测曲线与理想 预测曲线走形一致且贴近(图 5B)。上述结果均提 示模型预测效果良好,具有较高的预测价值。同 时,通过绘制建模组和验证组的决策曲线(图 6), 建模组临界概率值为 $0.01 \sim 0.62$, 验证组为 $0.01 \sim$ 0.81 , 患者均可实现临床获益, 说明模型在两组中 均取得了较好的临床运用效果。

模型在建模人群中进行 Hosmer-Lemeshow 检 验, 得到 $\chi^{2}=6.010, P>0.05$, 在验证人群中进 行 Hosmer-Lemeshow 检验, 得到 $\chi^{2}=4.215$, $P>0.05$, 提示模型拟合度良好。

\section{3 讨 论}

在以往的观念中, 乳腺癌被认为是局部区域 性疾病, 通过淋巴系统扩散, 所以 ALND一直是乳
腺癌患者腋窝管理的标准术式。20世纪 70 年代 的 NSABP-B04 随机对照试验结果表明, 早期乳腺

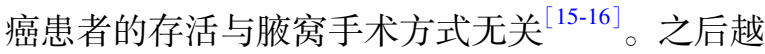
来越多的学者认为乳腺癌是全身性疾病, 控制局 部及全身复发有赖于综合治疗而不是区域淋巴结 清扫。因此, 从 20 世纪 90 年代开始, 对于早期乳 腺癌患者,SLNB 逐步取代了 $\mathrm{ALND}^{[15]}$ 。Z0011 试 验表明, 对于临床 T1 T2 期、1 2 个前哨淋巴结转 移、接受保乳手术和术后全乳放疗及全身辅助治 疗的患者,SLNB 和 ALND 治疗的患者局部控制率 和总存活率无明显差异 ${ }^{[3]}$ 。AMAROS 试验表明, 对于 $\mathrm{T} 1 \sim \mathrm{T} 2$ 期乳腺癌前哨淋巴结转移患者, 腋窝 放疗可替代 ALND, 且明显减少术后并发症的发 生 $^{[17]}$ 。各项研究表明, 即使前哨淋巴结阳性, 后 续进行全身治疗和放疗, 同样能得到与 ALND 类 

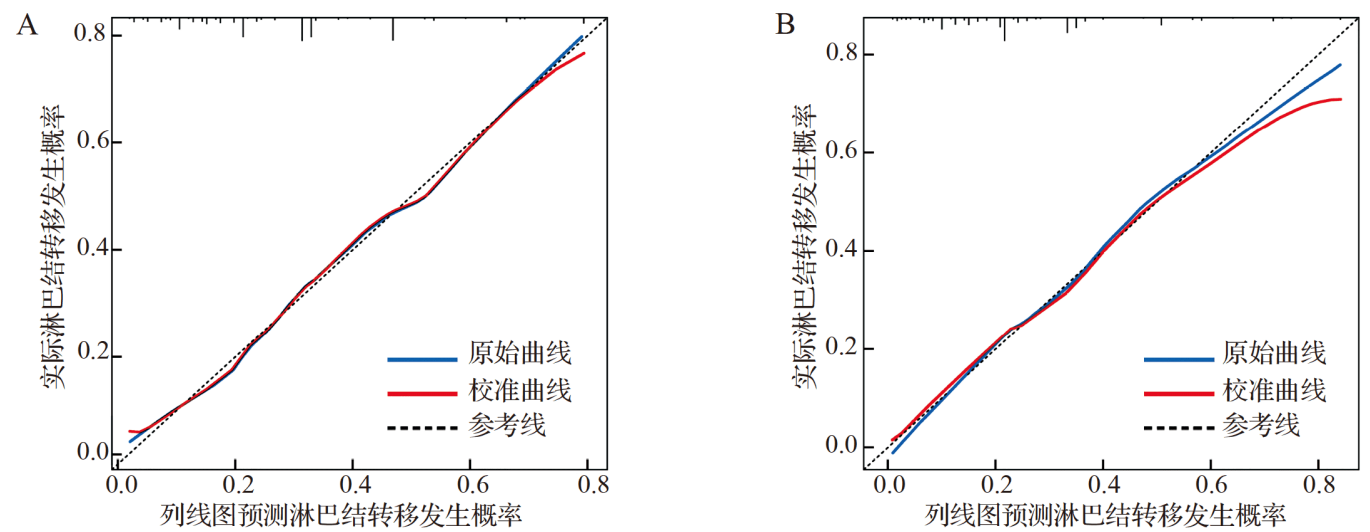

$A$ : 建模组; B : 验证组.

图 $5 \mathrm{~T} 1$ 期乳腺癌患者发生同侧腋窝淋巴结转移风险预测模型的校准曲线

Figure 5 Calibration curves of nomogram of the risk of ipsilateral axillary lymph node metastasis in patients with T1 breast cancer
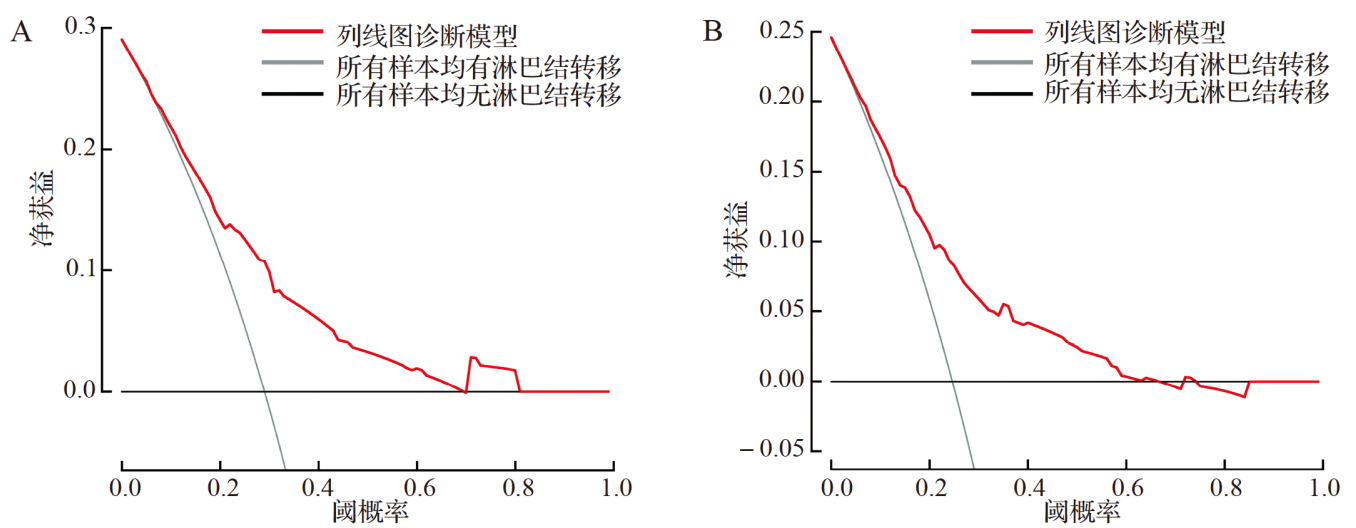

A: 建模组; B : 验证组.

图 $6 \mathrm{~T} 1$ 期乳腺癌患者发生同侧腋窝淋巴结转移风险预测模型的临床决策曲线

Figure 6 Decision curves of nomogram of the risk of ipsilateral axillary lymph node metastasis in T1 breast cancer

似的术后控制。这使得乳腺癌患者腋窝管理发生 了革命性改变, 越来越多的学者支持进一步减少 腋窝手术。SOUND 试验将超声评估腋窝淋巴结 阴性或细针穿刺证实淋巴结阴性的 T1 期乳腺癌 患者随机分为接受 SLNB 治疗和不接受 SLNB 治 疗两组, 研究两者对于淋巴结评估的有效性以及

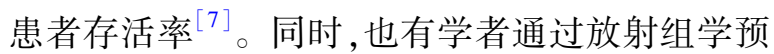
测乳腺癌腋窝淋巴结状态, 对预测结果为阴性的 患者免除 $\mathrm{SLNB}^{[6,8]}$ 。但无论是术前的淋巴结穿刺 还是放射组学的评估, 均对操作者和所用设备有 较高要求, 因此其临床推广受到限制。对于早期 乳腺癌患者发生同侧腋窝淋巴结转移的风险评估 是否可用临床更为可及且简便的方法来进行, 从 而避免进行腋窝手术, 本研究进行了一定探索。

本文资料显示, $\mathrm{T} 1$ 期乳腺癌患者淋巴结转移 的概率随着肿瘤增大而增加。多因素分析结果显
示, $\mathrm{T} 1 \mathrm{c}$ 患者 (肿瘤最大直径 $>1.0 \sim 2.0 \mathrm{~cm}$ ) 发生同 侧腋窝淋巴结转移的风险是 $\mathrm{T} 1 \mathrm{a}$ 患者 (肿瘤最大 直径介于 $0.1 \sim 0.5 \mathrm{~cm})$ 的 6.215 倍, 与之前的研究 结果一致 ${ }^{[18-19]}$ 。同时, 组织病理学分级和脉管癌 栓是淋巴结转移的独立影响因素, 这与多数文献 报道也是一致的 ${ }^{[20]}$ 。组织病理学分级越高, 肿瘤 分化程度越差, 发生淋巴结转移的风险越大 ${ }^{[21]}$, 组织病理学分级 3 级的患者发生淋巴结转移的风 险是 1 级患者的 5.183 倍。Ki-67 也是发生淋巴结 转移的独立预测因素。一般认为, Ki-67 高表达的 乳腺癌具有更强的侵袭性, 也更容易通过淋巴结 转移。大多数研究认为脉管癌栓是最强的淋巴结 转移风险因素 ${ }^{[22-24]}$, 其在本研究中同样显示出与 淋巴结转移高风险相关, 伴有脉管癌栓的浸润性 乳腺癌患者发生淋巴结转移的风险是不伴有脉管 癌栓浸润性乳腺癌患者的 5.156 倍, 说明原发肿 
瘤一旦伴有脉管癌栓, 肿瘤细胞已经有随脉管发 生转移的可能。

乳腺癌腋窝淋巴结转移与分子分型的关系一 直存在很大争议。一般来讲, 三阴性乳腺癌作为 恶性程度最高、侵袭性最强的乳腺癌类型, 最具有 发生转移的可能, 而 ER、PR 阳性的乳腺癌细胞分 化较好, 进展慢, 不易较早发生转移。然而, Crabb 等 ${ }^{25]}$ 对 4444 例早期乳腺癌患者的研究结果显示, Luminal 型乳腺癌较三阴性及 Her-2 过表达型更易 发生淋巴结转移, 提示具有激素受体阳性的乳腺 癌更倾向于通过淋巴管进行转移 ${ }^{[26]}$ 。本文资料 也印证了类似的现象, ER 阳性乳腺癌患者更易发 生淋巴结转移, 风险是 $\mathrm{ER}$ 阴性乳腺癌的 1.928 倍。但也有研究显示, 三阴性乳腺癌更易通过血 行转移, 故发生内脏转移的风险性高, 发生淋巴结 转移的风险性与其他分子分型相当 ${ }^{[26-27]}$ 。

本研究采用了列线图建立风险预测模型, 在 建模组和验证组中得到的 $\mathrm{C}$ 指数分别为 0.739 和 0.736 , 说明模型具有较好的预测性。同时, 模型 采用的均是临床常用且易于获取的数据, 模型更 易推广。此外, 本次建模组和验证组患者来自两 个不同地区不同中心, 数据具有明显差异, 但同样 取得了很好的验证结果, 说明模型的稳定性良好, 可以进行多中心推广。决策曲线也观察到了模型 的临床效益,具有临床推广价值。

综上所述, 原发肿瘤大小、组织病理学分级、 脉管癌栓、Ki-67、ER 状态对 T1 期乳腺癌患者发生 同侧腋窝淋巴结转移有重要影响。本研究通过上 述影响因素建立的列线图可有效预测腋窝淋巴结 转移风险, 帮助临床医生制订个体化的腋窝管理 决策。但本研究仍存在一定的局限性, 如病理数 据部分指标依靠人工判读, 存在一定误差, 再如回 顾性研究存在难以避免的潜在选择偏倚。因此, 本研究结论及模型有待在临床应用中进一步 验证。

\section{参考文献}

［1］郑荣寿, 孙可欣,张思维, 等. 2015 年中国恶性肿瘤 流行情况分析 $[J]$. 中华肿瘤杂志, 2019,41(1): 1928. DOI: 10. 3760/cma. j. issn. 0253-3766. 2019. 01. 005 .

ZHENG Rongshou,SUN Kexin,ZHANG Siwei,et al. Report of cancer epidemiology in China,2015[J]. Chinese Journal of Oncology, 2019,41(1):19-28.
DOI: 10. 3760/cma. j. issn. 0253-3766. 2019. 01. 005. (in Chinese)

[2] VERONESI P, CORSO G. Standard and controversies in sentinel node in breast cancer patients $[\mathrm{J}]$. Breast, 2019,48 Suppl 1:S53-S56. DOI:10. 1016/S09609776(19)31124-5.

[3] GIULIANO A E, BALLMAN K V, MCCALL L, et al. Effect of axillary dissection vs no axillary dissection on 10-year overall survival among women with invasive breast cancer and sentinel node metastasis $[\mathrm{J}]$. JAMA, 2017,318(10):918-926. DOI: 10. 1001/jama. 2017. 11470 .

[4] BUNDRED N J, BARNES N L P, RUTGERS E, et al. Is axillary lymph node clearance required in node-positive breast cancer? [J]. Nat Rev Clin Oncol, 2015, 12(1): 55-61. DOI:10. 1038/nrclinonc. 2014. 188.

[5] BEEK M A, VERHEUVEL N C, LUITEN E J T, et al. Two decades of axillary management in breast cancer [J]. Br J Surg, 2015,102(13): 1658-1664. DOI: 10. 1002/bjs. 9955.

[6] ZHENG X, YAO Z, HUANG Y, et al. Deep learning radiomics can predict axillary lymph node status in early-stage breast cancer[J]. Nat Commun, 2020,11 (1) : 1236. DOI : 10. 1038/s41467-020-15027-z.

[7] GENTILINI O, BOTTERI E, DADDA P, et al. Physical function of the upper limb after breast cancer surgery. Results from the SOUND (Sentinel node vs. Observation after axillary Ultra-souND) $\operatorname{trial}[\mathrm{J}]$. Eur J Surgical Oncology, 2016,42(5) : 685-689. DOI : 10. 1016/j. ejso. 2016. 01. 020.

[8] QIU S Q, AARNINK M, VAN MAAREN M C, et al. Validation and update of a lymph node metastasis prediction model for breast cancer $[J]$. Eur J Surgical Oncology, 2018,44(5):700-707. DOI:10. 1016/j. ejso. 2017. 12. 008.

[9] GIULIANO A E, EDGE S B, HORTOBAGYI G N. Eighth edition of the AJCC cancer staging manual: breast cancer[J]. Ann Surg Oncol, 2018,25(7): 1783-1785. DOI: 10. 1245/s10434-018-6486-6.

[10] GOLDHIRSCH A, WINER E P, COATES A S, et al. Personalizing the treatment of women with early breast cancer :highlights of the St Gallen International Expert Consensus on the Primary Therapy of Early Breast Cancer 2013[J]. Ann Oncology, 2013,24(9):22062223. DOI: $10.1093 /$ annonc/mdt303.

[11 COATES A S, WINER E P, GOLDHIRSCH A, et al. Tailoring therapies-improving the management of early breast cancer:St Gallen International Expert Consensus on the Primary Therapy of Early Breast Cancer 2015 [J]. Ann Oncology, 2015,26(8): 15331546. DOI: $10.1093 /$ annonc/mdv221.

[12］梅 放,柳剑英,薛卫成. 浸润性乳腺癌的组织学 分级:Nottingham 组织学分级系统 $[\mathrm{J}]$. 中华病理学 
杂志 $, 2019,48(8): 659-664$. DOI: 10. 3760/cma. j. issn. 0529-5807. 2019. 08. 019.

MEI Fang, LIU Jianying, XUE Weicheng. Histological grading of invasive breast carcinoma: Nottingham histological grading system $[\mathrm{J}]$. Chinese Journal of Pathology ,2019,48(8) :659-664. DOI: 10.3760/ cma. j. issn. 0529-5807. 2019. 08. 019. (in Chinese)

[13] 王一澎, 张 扬, 王仲照, 等. 2108 例 $\mathrm{T}_{-}(1$ 2) 期乳 腺癌患者腋窝淋巴结转移的相关因素分析 $[\mathrm{J}]$. 癌 症进展, 2016,14(6):530-534. DOI: 10.11877/j. issn. 1672-1535. 2016. 14. 06. 09.

WANG Yipeng,ZHANG Yang, WANG Zhongzhao, et al. Correlation between clinicopathological characteris tics and axillary lymph nodes metastasis in 2108 patients with T1-2 breast carcinoma $[\mathrm{J}]$. Oncology Progress , 2016,14(6):530-534. DOI: 10. 11877/j. issn. 1672-1535. 2016. 14. 06. 09. (in Chinese)

[14] 焦得闯, 乔江华, 朱久俊, 等. $\mathrm{T} 1$ 期乳腺癌腋窝淋巴 结转移及乳腺癌特异生存的影响因素分析 $[\mathrm{J}]$. 中 华医学杂志, 2018,98(40):3258-3262. DOI: 10. 3760/cma. j. issn. 0376-2491. 2018. 40. 009.

JIAO Dechuang, QIAO Jianghua,ZHU Jiujun, et al. Analysis of factors influencing the axillary lymph node metastasis and breast cancer-specific survival in patients with T1 breast cancer $[J]$. National Medical Journal of China, 2018,98(40):32583262. DOI: 10. 3760/cma. j. issn. 0376-2491. 2018. 40. 009. (in Chinese)

[15] RIEDEL F, HEIL J, GOLATTA M, et al. Changes of breast and axillary surgery patterns in patients with primary breast cancer during the past decade $[\mathrm{J}]$. Arch Gynecol Obstet, 2019,299(4):1043-1053. DOI: $10.1007 / \mathrm{s} 00404-018-4982-3$.

[16] GERA R, KASEM A, MOKBEL K. Can complete axillary node dissection be safely omitted in patients with early breast cancer when the sentinel node biopsy is positive for malignancy? An update for clinical practice[J]. In Vivo, 2018,32(6): 1301-1307. DOI: 10. 21873/invivo. 11380.

[17] DONKER M, VAN TIENHOVEN G, STRAVER M E, et al. Radiotherapy or surgery of the axilla after a positive sentinel node in breast cancer (EORTC 10981-22023 AMAROS): a randomised, multicentre, open-label, phase 3 non-inferiority $\operatorname{trial}[\mathrm{J}]$. Lancet Oncology, 2014,15(12): 1303-1310. DOI:10. 1016/ S1470-2045(14)70460-7.

[18] LOZA C M, MANDÓ P, PONCE C, et al. Predictive factors for non-sentinel lymph node metastasis in patients with ACOSOG Z0011 criteria[J]. Breast Care, 2018,13(6) : 434-438. DOI : 10. 1159/000488277.

[19] YUN S J, SOHN Y M, SEO M. Risk stratification for axillary lymph node metastases in breast cancer patients $[\mathrm{J}]$. Ultrasound Q, 2017,33(1):15-22. DOI: 10. 1097/RUQ. 0000000000000249.

[20] YOSHIHARA E, SMEETS A, LAENEN A, et al. Predictors of axillary lymph node metastases in early breast cancer and their applicability in clinical practice $[\mathrm{J}]$. Breast, 2013,22(3):357-361. DOI : 10. 1016/j. breast. 2012.09. 003.

[21] TSENG H S, CHEN L S, KUO S J, et al. Tumor characteristics of breast cancer in predicting axillary lymph node metastasis[J]. Med Sci Monit, 2014, 20:1155-1161. DOI:10. 12659/MSM. 890491.

[22] CHUNG M J, LEE J H, KIM S H, et al. Simple prediction model of axillary lymph node positivity after analyzing molecular and clinical factors in early breast cancer $[\mathrm{J} / \mathrm{OL}]$. Medicine, 2016,95(20): e3689. DOI : $10.1097 /$ MD. 0000000000003689.

[23] SHAYAN R, INDER R, KARNEZIS T, et al. Tumor location and nature of lymphatic vessels are key determinants of cancer metastasis $[\mathrm{J}]$. Clin Exp Metastasis, 2013,30(3):345-356. DOI: 10. 1007/ s10585-012-9541-x.

[24] YU H, ZHANG S, ZHANG R, et al. The role of VEGFC/D and Flt-4 in the lymphatic metastasis of earlystage invasive cervical carcinoma $[J]$. J Exp Clin Cancer Res, 2009,28:98. DOI:10. 1186/1756-996628-98.

[25 ] CRABB S J, CHEANG M C U, LEUNG S, et al. Basal breast cancer molecular subtype predicts for lower incidence of axillary lymph node metastases in primary breast cancer $[\mathrm{J}]$. Clin Breast Cancer, 2008,8(3): 249-256. DOI: 10. 3816/CBC. 2008. n. 028.

[26] RODRÍGUEZ-PINILLA S M, SARRIÓ D, HONRADO E, et al. Prognostic significance of basal-like phenotype and fascin expression in node-negative invasive breast carcinomas $[\mathrm{J}]$. Clin Cancer Res, 2006,12(5): 1533-1539. DOI:10. 1158/1078-0432. CCR-05-2281.

[27] MADSEN E V E, ELIAS S G, VAN DALEN T, et al. Predictive factors of isolated tumor cells and micrometastases in axillary lymph nodes in breast cancer $[\mathrm{J}]$. Breast, 2013,22(5):748-752. DOI: 10. 1016/j. breast. 2012. 12. 013 .

[本文编辑 沈 敏 余 方] 